\title{
FACTOR INTENSITY AND SITE GEOLOGY AS DETERMINANTS OF RETURNS TO SCALE IN COAL MINING
}

\begin{abstract}
Increasing returns to scale (RTS) is frequently postulated as affecting productivity in surface coal mining. However, it is not clear whether increased capital intensity or increased output is the relevant phenomenon. A ray-homothetic production function that incorporates the capital-labor mix and fixed site geology into the scale elasticity is presented and estimated with a micro (mine level) dataset. The results indicate that higher capital intensity contributes to higher RTS for some types of capital equipment, but not all. On the average increasing RTS was found, with few mines approaching optimal scale.
\end{abstract}

Gale A. Boyd*

\section{Introduction}

$\mathrm{T}$ HE literature of coal mining productivity contains many references to returns to scale as a factor in strip mining productivity. ${ }^{1}$ However, different analysts use different definitions of "scale," and consequently their results are mixed. Some analysts associate returns to scale with larger pieces of capital equipment ${ }^{2}$ others use the more traditional economic notion of output volume and the scale elasticity; ${ }^{3}$ others simply relate output volume to labor productivity. ${ }^{4}$ It may be true that developments in large pieces of earth-moving equipment have been implemented at surface mines with large output volume, but this does not necessarily imply increasing returns to this particular capital input. This confusion in the mining

Received for publication November 13, 1985. Revision accepted for publication June 18, 1986.

*Argonne National Laboratory.

This paper follows substantially the results of my $\mathrm{Ph} . \mathrm{D}$. thesis. I would like to thank my wife, Emily Boyd; my mother, Norma Parrish; my chairman. Rolf Färe; and my committee for their patience and assistance during my graduate work. The empirical work contained herein would not have been possible without the support of the Coal Technology Laboratory at Southern Illinois University-Carbondale, who provided the data. Work on this paper was partially supported by the U.S. Department of Energy, Office of Fossil Energy, under Contract W-31-109-Eng-38. I would also like to acknowledge the helpful comments of Ken Rose and two anonymous referees in the preparation of this paper. Any errors remaining are the sole responsibility of the author.

${ }^{1}$ For a complete discussion, see Boyd (1984).

See Hill (1980), Lakhani (1980), Murray (1980), and Zimmerman (1981).

${ }^{3}$ See Färe and Yoon (1983), Kruvant, Moody, and Valentine (1982), Maddala (1965), and Myers and Färe (1982).

${ }^{4}$ See Baker (1979) and Malhotra (1975). literature in the use of the term "scale," coupled with the more general observation that large firms (not just large mines) rarely have the same capital-labor mix as their smaller counterparts, leads to a hypothesis that a different capital-labor mix yields different economies of scale.

The application of ray-homothetic production functions leads to an easily testable hypothesis on the impact of input mix to economies of scale. Additionally, these functions are more general than their homothetic namesakes. Färe (1975) has shown that they do not generate linear expansion paths, convex isoquants, or exhibit strong disposability of inputs. The properties of convexity and strong disposability are necessary for a dual, cost function analysis of the production structure. If the true underlying production function is rayhomothetic, the dual approach is inappropriate, therefore these functions are a desirable tool for productivity analysis in general and in particular when input mix is believed to be an important determinant of economies of scale. ${ }^{5}$

Newcombe (1978) illustrates that geological conditions are a key determinant of strip mine productivity. The decision to open a mine and the scale of that mine critically depend on the site geology. ${ }^{6}$ By appropriate choice of homothetic transformation, it is possible to model another significant phenomenon in strip mining, capacity constraints that arise from the mine site geology. ${ }^{7}$ The mine site geology is, by nature, permanently fixed to the firm. Key geological descriptors like the coal seam thickness or reserves determine the

\footnotetext{
${ }^{5}$ One significant drawback with a production function approach, however, is that endogeneity of the input variables will result in inconsistent estimates. Since the cost function is the principal way around this problem, we are faced with a methodological tradeoff between an undesirable statistical procedure and an undesirable theoretical procedure. The choice in this case is made in favor of the more satisfying theoretical method

${ }^{6}$ For a discussion of mine site engineering and site geology, see Skelly and Loy (1975).

${ }^{7}$ Capacity bounds are often referred to but rarely explicitly modeled. The approach used here is applicable to any firm with fixed inputs that imply an upper bound on output.
} 
volume of coal that may be mined in a given period. The need for maintaining overburden piles while the coal is removed from the seam creates congestion in an engineering sense and in the sense of the law of diminishing returns. The law of diminishing returns, due to the presence of these fixed factors, may be modeled by a modified arctangent homothetic transformation. Alternative treatments of diminishing returns in production functions are discussed by Shephard and Färe (1974) but do not lend themselves to analysis of returns to scale in the variable factors. Additionally, modeling fixed factors in an appropriate homothetic transformation makes it possible to define and compute optimal scale for each mine.

The empirical example estimated is for the midwest mining region. The main result of this analysis agrees with the notion that more intensive use of capital equipment vis à vis labor inputs, specifically the primary earth mover, contributes to increased returns to scale. Increasing returns to the primary earth mover alone is not found. The production function presented below fits the data well and passes a test for functional specification.

Section II reviews the theory of ray-homotheticity and presents the Ray Cobb Douglas (RCD) production function and a homothetic transformation to model capacity bounds. Section III combines the RCD with the arctangent homothetic transformation and estimates the model for the coal strip-mining industry. Section IV discusses the scale elasticity estimates and computes optimal scale from the estimates in section III.

\section{Functional Form of the Model}

The class of ray-homogeneous functions was introduced by Eichhorn (1969). These functions are homogeneous of degree $A(X /|X|)$, where $A$ is a positive valued function which is homogeneous of degree zero in $X$.

$$
g(\lambda X)=\lambda^{A(X /|X|)} g(X) .
$$

The function $g$ is homogeneous of degree $A(X /|X|)$ along each ray in input space.

Ray-homothetic functions may be defined analogously to homothetic functions. A ray-homothetic function is a monotone transformation, $h^{-1}$, of a ray-homogeneous "kernel" function, $g(X)$.

$$
f(x)=h^{-1}(g(X)) \text {. }
$$

Discussion of choice of an appropriate homothetic transformation is deferred to the end of this section.

The properties of the ray-homogeneous class are examined by Goldman and Shephard (1972), who show that this type of function exhibits either convexity or strong disposability only when the function reduces to simple homogeneous; i.e., $A(X /|X|)$ is constant. A monotone transformation only reorders the isoquants of a ray-homogeneous function. It does not affect its curvature properties (convexity, disposability). Thus, the result obtained by Goldman and Shephard is true for ray-homothetic functions as well. Färe (1975) shows that a ray-homothetic function satisfies these stronger assumptions if it is a simple homothetic function.

The functional form of the kernel which is presented here is the ray-homogeneous CobbDouglas (RCD),

$$
g(X)=e^{\delta} \prod_{i=1}^{n} X_{i}^{\alpha_{i}+a(X /|X|)} .
$$

The function $g(X)$ is ray-homogeneous of degree $A(X /|X|)$, where

$$
A(X /|X|)=\sum_{i=1}^{n}\left(\alpha_{i}+a(X /|X|)\right) .
$$

The function $a$ is real valued and homogeneous of degree zero in $X$. This general form was introduced by Färe, Jansson and Lovell (1981). One should note that the only requirement on $a(X /|X|)$ is that it be homogeneous of degree zero in $X$. It is not necessary to choose an explicit norm on $X$. The notation $a(X /|X|)$ and $A(X /|X|)$ is used to make it clear that the function must be homogeneous of degree zero in $X$.

The form of $a(X /|X|)$ that is used here ${ }^{8}$ is

$$
a(X /|X|)=\sum_{j=2}^{n} \beta_{j} X_{j} / X_{1} \text {. - }
$$

The "norm" is taken to be the first input in the $X$ vector. More specifically, $X_{1}$ will be labor. If the other inputs are capital equipment, then the capital-labor ratios will affect the degree of homo-

\footnotetext{
${ }^{8}$ The linear form for the degree of ray-homogeneity, $A(X /|X|)$, may be thought of as an approximation to a more general relationship. Although it is unreasonable for $A(X /|X|)$ $\rightarrow \infty$ as $x_{i} / x_{1} \rightarrow \infty$, the linear relationship should be a reasonable approximation for the observed range of $X_{i} / X_{1}$, $i=2, \ldots, n$. Furthermore, we expect $A(X /|X|)>0$ for the observed range of $X_{i} / X_{1}$.
} 
geneity. This formulation also allows for relatively simple derivation of marginal products. A nonlinear $a(X /|X|)$ or a more complicated "norm," euclidean for example, would make the derivation of marginal products unnecessarily complex. ${ }^{9}$ The functional form presented here is not flexible in the conventional sense of being able to provide a second-order approximation to an arbitrary production function, but may be seen as "flexible" in its representation of scale economies, since it generalizes the degree of homogeneity of the CobbDouglas form.

The behavior of the scale elasticity, $E$, with respect to output, $u$, as inputs are scaled along some path is important to empirical work. In particular, it is desirable for the production function to exhibit some notion of a capacity bound. This is especially true of micro (mine level) production functions. In order for the scale elasticity to reflect this, the functional form of $h$ and hence $E$ must exhibit certain properties.

Let the capacity limit be denoted as $U$, then

$$
\lim _{\lambda \rightarrow \infty} h^{-1}(g(\lambda X))=U, h^{-1}(g(\lambda X))<U \text {. }
$$

That is, as inputs are scaled, output approaches the capacity limit from below. This is what is meant by capacity limit. An alternative way to express a capacity limit is

$$
\lim _{u \rightarrow U} E(u, X)=0
$$

when scaling is radial. In addition, $E$ should decline monotonically, from values above one to zero, as $u$ ranges from zero to $U$. This models the assumption of initially increasing and then decreasing RTS as output exceeds the optimal scale.

The homothetic transformation used here is a modification of the one introduced by Waage (1974). Let

$$
h(u)=\tan \left(u / a_{0}\right) .
$$

This has a capacity limit of $a_{0} \pi / 2$, and has a much simpler scale elasticity than the original form introduced by Waage.

$$
\begin{aligned}
& E(u, X) \\
& \quad=A(X /|X|) \sin \left(u / a_{0}\right) \cos \left(u / a_{0}\right)\left(a_{0} / u\right) \\
& \quad=A(X /|X|) \sin \left(2 u / a_{0}\right)\left(a_{0} / 2 u\right) .
\end{aligned}
$$

\footnotetext{
${ }^{9}$ For more detailed results on marginal products, see Boyd (1984).
}

The behavior of (9) for small $u$ can be seen by writing

$$
\begin{aligned}
\lim _{u \rightarrow 0} E(u, X)= & A(X /|X|) \\
& \times \lim _{u \rightarrow 0} \sin \left(2 u / a_{0}\right) /\left(2 u / a_{0}\right) .
\end{aligned}
$$

Denoting $2 u / a_{0}=v$, then

$$
\begin{aligned}
\lim _{u \rightarrow 0} E(u, X) & =A(X /|X|) \lim _{v \rightarrow 0} \sin (v) / v \\
& =A(X /|X|) \cdot 1 .
\end{aligned}
$$

Hence, (9) may exhibit initially increasing RTS only when $A(X /|X|)>1$.

The capacity bound of a plant is determined by the level of fixed factors of production. What is meant by fixed and variable depends, in part, on the time period one is concerned with. Let us assume here that the factors of production, $X$, can be partitioned into fixed and variable components, i.e., $X=(z, x)$, where $z$ is a subvector of fixed factors and $x$ is the remaining $n$-dimensional subvector of variable factors. The capacity bound can now be expressed as $u=c(z)$, or in the case of the homothetic transformation in (8), $a_{0}=c(z)$, since $a_{0}$ is the parameter that determines the capacity bound.

Mak (1980) provides the theoretical connection between the partition above and the ray-homogeneous kernel function represented by (1). He generalizes ray-homogeneous functions to path-homogeneous ${ }^{10}$ functions, where any arbitrary path in input space may be the scaling path. In our case, the scaling path is in the sub-space of variable factors, i.e., $\lambda X=(z, \lambda x)$. The resulting function is path-homogeneous for partial (variable) factor scaling. Since the path is linear in the variable factors, the term ray-homogeneous and ray-homothetic can be used loosely within this context without confusion. Since the change to variable factor scaling does not affect the theory, the only notational change will be to use small $x$ to denote variable factors.

Within the context of strip mining, the geological characteristics of the mine are the fixed factors. Let

$$
c(z)=e^{\delta_{1}} \prod_{i=1}^{k} z_{i}^{\gamma_{i}}
$$

\footnotetext{
${ }^{10}$ Author's term.
} 
where $z^{i}, i=1, \ldots, k$, are the geological characteristics of the mine and $\gamma_{i}, i=1, \ldots, k$ are parameters. The homothetic transformation is a function of both output and fixed geological characteristics. To see this, combining (11) with (8) yields the modified arctangent form,

$$
h(u, z)=\tan \left[u /\left(e^{\delta_{1}} \prod_{i=1}^{k} z_{i}^{\gamma_{i}}\right)\right] .
$$

Capacity is expressed as a function of the fixed inputs, and hence it will vary from mine to mine.

Combining (3), (5), and (12) yields a production function with a scale elasticity with the desired properties.

$$
\begin{aligned}
u= & e^{\delta_{1}} \prod_{i=1}^{k} z_{i}^{\gamma_{i}} \\
& \times \tan ^{-1}\left[e^{\delta} \prod_{i=1}^{n} x_{i}^{\left(\alpha_{i}+\sum_{j=2}^{n} \beta_{j} x_{j} / x_{1}\right)}\right] .
\end{aligned}
$$

The scale elasticity for (13) is

$$
\begin{aligned}
E(u, X)= & {\left[\sum_{i=1}^{n} \alpha_{i}+n \sum_{j=2}^{n} \beta_{j} x_{j} / x_{1}\right] \sin \left(\frac{2 u}{c(z)}\right) } \\
& \times \frac{c(z)}{2 u} .
\end{aligned}
$$

This form is the ray-homothetic Cobb-Douglas (RHCD), for partial factor scaling. The homothetic transformation is the modified arctangent form.

\section{Estimation}

To illustrate the use of this model, it is applied to the surface coal mining industry in the Illinois Basin mining region. The data are derived from an Energy Information Administration survey ${ }^{11}$ and are documented by Boyd (1984). Since the survey identifies actual mining operations, confidentiality is maintained to avoid disclosure of company information. The data used here are annual, covering the period 1975-78. The survey gives information on tons of coal shipped, labor, capital equipment, and geological characteristics. Other relevant information available includes the number of days the mine operated. The variable inputs

\footnotetext{
${ }^{11}$ Summary statistics may be found in Bituminous Coal and Lignite Production and Mine Operations, various years.
}

are denoted by

$x_{1}=$ labor, in hundreds of person hours;

$x_{2}=$ primary earth moving capital;

$x_{3}=$ reclamation capital; and

$x_{4}=$ other capital.

Capital flows are in thousands of dollars and are adjusted for utilization. The number of days the mine was open is the basis for the utilization measure. Geological characteristics are denoted by

$z_{1}=$ overburden thickness, in feet, and

$z_{2}=$ average seam thickness, in inches.

Output is denoted by

$u=$ coal shipped, in millions of tons.

Appending a statistical error term to (13) yields the estimating equation,

$$
\begin{aligned}
u= & e^{\delta_{1}} z_{1}^{\gamma_{1}} z_{2}^{\gamma_{2}} \\
& \times \tan ^{-1}\left[e^{\delta_{2}} \prod_{i=1}^{4} x_{i}^{\alpha_{i}+\left(\beta_{2} x_{2}+\beta_{3} x_{3}+\beta_{4} x_{4}\right) / x_{1}}\right]+\epsilon
\end{aligned}
$$

where greek letters denote parameters to be estimated. Following Zellner, Kmenta, and Dréze (1966), we now assume that $E(\epsilon)=0, E\left(\epsilon^{\prime} \epsilon\right)=$ $\sigma^{2} I$, the operators of the mines included in the data set maximize expected profit, and the errors are normally distributed so that maximum likelihood techniques apply. Jennrich (1969) shows that a method which is asymptotically equivalent to maximum likelihood is nonlinear least squares (NLS). It is assumed that the sample size is sufficient to claim asymptotic properties.

Plots of residuals against the number of days the mine operated suggested that the errors are heteroscedastic. Several tests for heteroscedasticity were performed and weighted NLS was applied to estimate (15). Additional information on the estimation procedure is available from the author on request.

The results for the weighted NLS model are presented in table 1 . The value of $R^{2}, 0.908$, is very high for a cross-section data set. All parameters in the model are significant in a two-tailed test at the $95 \%$ confidence level, except the four indicated. The significance of $\beta_{2}$ implies a rejection of the simple homothetic $C D$ form, supporting the hypothesis that factor intensity has an impact on RTS. The degree of homogeneity is not constant, but depends upon input mix. 
TABLE 1.-WNLS RESULTS

\begin{tabular}{lcr}
\hline \hline Parameter & Estimate & $t$-ratio \\
\hline$\delta_{1}{ }^{\mathrm{a}}$ & 1.47 & 1.9 \\
$\delta_{2}$ & -10.7 & -10.6 \\
$\gamma_{1}$ & -0.162 & -2.2 \\
$\gamma_{2}$ & 0.366 & 5.8 \\
$\alpha_{1}{ }{ }^{b}$ & 1.03 & 8.5 \\
$\alpha_{2}$ & -0.0637 & -0.5 \\
$\alpha_{3}$ & -0.256 & -3.6 \\
$\alpha_{4}$ & 0.326 & 3.0 \\
$\beta_{2}$ & 24.2 & 4.3 \\
$\beta_{3}{ }^{\mathrm{c}}$ & 38.7 & 1.4 \\
$\beta_{4}{ }^{\mathrm{c}}$ & -65.5 & 1.6 \\
$R^{2}=.908$ & &
\end{tabular}

${ }^{a}$ Significant at the $10 \%$ level.

${ }^{\mathrm{b}}$ Not significant.

${ }^{c}$ Significant at the $10 \%$ level in one-tailed test.

One test employed for heteroscedasticity is sensitive to functional misspecification as well as the failure of $\epsilon$ to satisfy homoscedasticity. If the true model is actually

$$
u_{t}=G\left(Z_{t}: \theta^{*}\right)
$$

then the test for heteroscedasticity would fail. Functional specification is also an interesting question in its own right. Fortunately, the existence of two estimators of the specified model, (15), makes a test for functional specification relatively easy to compute.

White (1980) proposes a test of functional specification utilizing the weighted and unweighted NLS estimates. This test is in a general class of estimators proposed by Hausman (1978). What is required is a consistent estimator of $\theta$ and a second estimator which is asymptotically efficient under $H_{0}$. In this case they are the NLS and the WNLS estimators, respectively. Denoting the NLS estimates by $\theta^{1}$ and the WNLS estimates by $\theta^{2}$ with $V^{1}$ and $V^{2}$ the respective unconditional covariance estimates, we have the test statistic

$$
n\left(\theta^{1}-\theta^{2}\right)^{\prime}\left[V^{1}-V^{2}\right]^{-1}\left(\theta^{1}-\theta^{2}\right) \stackrel{A}{\sim} \chi_{k}^{2} \text {. }
$$

Computing the above test statistic from the two sets of estimates yields a test statistic of 14.8. The critical value for $\chi_{11}^{2}$ is 19.6 at the $5 \%$ level. Thus, we accept the null hypothesis of no misspecification.

\section{Results}

Combining equations (11) and (14) yields the equation for the scale elasticity:

$$
\begin{aligned}
E(u, X)= & {\left[\sum_{i=1}^{4} \alpha_{i}+4 \sum_{j=2}^{4} \beta_{j} x_{j} / x_{1}\right] \sin \left(\frac{2 u}{z_{1}^{\gamma_{1}} z_{2}^{\gamma_{2}}}\right) } \\
& \times \frac{z_{1}^{\gamma_{1}} z_{2}^{\gamma_{2}}}{2 u} .
\end{aligned}
$$

The scale elasticity is not constant, but depends upon fixed factors, output levels, and capital-labor ratios.

The significance of $\hat{\beta}_{2}$ implies that higher capital intensity in the primary earth mover increases RTS. This result is consistent with the observations in the literature associating scale economies with the size of the primary earth mover, but makes that connection explicit. Increasing returns to the primary earth mover alone was not supported by these estimates. The tendency for other studies estimating traditional production functions and obtaining slightly increasing, but not significant economies of scale is not surprising when taken in light of the dependence of $E$ on this important capital-labor ratio, which varies from mine to mine.

Applying (18) to all observations in the data set yields an elasticity that varies from 0.77 to 3.0. The mean value is 1.24 . Only eleven observations had scale elasticities less than unity. To illustrate the variation in the scale elasticity by mine size, frequencies by output level and scale elasticity are shown in table 2.

TABle 2.-Mine Frequencies by Size, ${ }^{a} u$, ANd SCAle Elasticity $E$

\begin{tabular}{rrrrrrr}
\hline \hline & $u<.05$ & $.05<u<.1$ & $.1<u<.5$ & $.5<u<1$ & $1<u<1.5$ & $1.5<u$ \\
\hline \multicolumn{1}{c}{$E<1.1$} & 5 & 5 & 19 & 5 & 3 & 6 \\
$1.1<E<1.2$ & 8 & 10 & 31 & 14 & 10 & 12 \\
$1.2<E<1.3$ & 7 & 5 & 15 & 9 & 4 & 6 \\
$1.3<E<1.4$ & 10 & 2 & 5 & 2 & 2 & 3 \\
$1.4<E<1.5$ & 9 & 1 & 1 & 1 & 1 & 1 \\
$1.5<E$ & 18 & 1 & 2 & 0 & 0 & 0 \\
\hline
\end{tabular}

${ }^{\mathrm{a}}$ Units $=$ millions of tons shipped. 
As observed in section II, the scale elasticity may exhibit initially increasing, constant, and finally decreasing RTS as $u \rightarrow U$ only when $A(x /|x|)>1$. Optimal scale is obtained when $E(u, X)=1$. When $A(x /|x|)<1$ there does not exist an optimal scale. This is the case for only eleven observations in the data set.

To obtain the optimal scale, let $v=u / e^{\delta_{1}} z_{1}^{\gamma_{1}} z_{2}^{\gamma_{2}}$ and solve

$$
A(x /|x|) \sin (v) \cos (v) / v=1 \text {. }
$$

This equation is nonlinear in $v$ and has no analytical solution. Newton's method, an iterative method for solving nonlinear equations, may be applied to obtain a solution.

Optimal scale ranges from 0.65 to 10.3 million tons per year. The mean value is 4.8 . Few mines approach the optimum. The theoretical capacity bound shows similar variation. The mean value is 13.8, with lower and upper bounds of 9.3 and 23.5 , respectively. Optimal scale tends to be much lower than the capacity bound. That is because the capacity bound is the theoretically maximum output level. This is not an engineering notion of "practical capacity." One would expect "practical capacity" to be closer to optimal scale. The capacity bound may also be interpreted as a measure of the productivity of the fixed geological conditions, since the fact that these key productive inputs are fixed is what gives rise to capacity bounds.

Fixed inputs and capital intensity clearly have a role in determining the nature of returns to scale. The production function presented here demonstrates that the effects of these factors can be modeled and parametrically tested. Since the scale elasticity exhibits increasing, constant, and decreasing returns, as output rises, it is possible to compute optimal scale. Although the model is nonlinear, improvements in computational methods and computers have removed this as an impediment to the useful application of this empirical tool. In the example presented, higher capital intensity in the primary earth mover has a significant impact on returns to scale. This may be seen as supporting two apparently different points of view in the mining productivity literature: increasing returns to the primary earth mover and increasing returns to scale.

\section{REFERENCES}

Baker, Joe, "Determinants of Coal Mine Labor Productivity Change," Oak Ridge Associated Universities, DOE/IR/0056 (1979).
Boyd, Gale, "Scale and Productivity in Coal Strip Mining," $\mathrm{Ph} . \mathrm{D}$. thesis, Southern Illinois University-Carbondale (1984).

Eichhorn, W., "Eine Verallgemeinerung des Begriffs der Homogenen Produktionfunktion," Unternehmensforschung 13 (2) (1969), 99-109.

Färe, Rolf, "A Note on Ray-homogeneous and Ray-homothetic Production Functions," The Swedish Journal of Economics 77 (1975), 366-372.

Färe, Rolf, Lief Jansson, and C. Knox Lovell, "Modelling Scale Economies with Ray-homothetic Production Functions," this REVIEW 67 (4) (1985), 624-629.

Färe, Rolf, and Bong Yoon, "Returns to Scale in U.S. Surface Mining of Coal," Southern Illinois University, Department of Economics Discussion Paper No. 83-10 (1983).

Goldman, Stephen, and Ronald Shephard, "On Eichhorn's Generalization of Homogeneous Production Functions," Zeitschrift für Operations Research 16 (1972), 215-219.

Hausman, Jerry, "Specification Tests in Econometrics," Econometrica 46 (6) (1978), 1251-1271.

Hill, Forrest E., "Causes for the Productivity Decline in U.S. Coal Mining," Mining Congress Journal 66 (9) (1980), 32-37.

Jennrich, Robert, "Asymptotic Properties of Non-linear Least Squares Estimators," Annals of Mathematical Statistics 40 (2) (1969), 633-643.

Kruvant, William J., Carlisle Moody, and Patrick Valentine, "Sources of Productivity Decline in U.S. Coal Mining, 1972-1977," The Energy Journal 3 (3) (1982), 53-70.

Lakhani, Hyder, "Economics of Technical Change in U.S. Coal Mines, 1951-75," Energy 5 (1) (1980), 217-230.

Maddala, G. S., "Productivity and Technical Change in the Bituminous Coal Industry 1919-54," The Journal of Political Economy 73 (4) (1965).

Mak, King-Tim, "General Homothetic Production Correspondences," Operations Research Center, University of California-Berkeley (1980).

Malhotra, Ramesh, "Factors Responsible for Variation in Productivity in Illinois Coal Mines," Illinois Mineral Note 60 (Urbana: Illinois State Geological Survey, 1975), 4-9.

Murray R., "Productivity Problems in Western Surface Coal Mining," Mining Congress Journal 66 (8) (1980), 43-48.

Myers, John, and Rolf Färe, Productivity in Strip Mining of Coal, Final Report, Coal Extraction and Utilization Research Center, Southern Illinois University-Carbondale (1982).

Newcombe, R., "The Geologic and Statistical Characteristics of Coal Supply Models," Materials and Society 2 (1978), 45-57.

Shephard, Ronald, and Rolf Färe, "The Law of Diminishing Returns," Zeitschrift fur Nationalökonomie 34 (1974), 69-90.

Skelley and Loy Consultants, Economic Engineering Analysis of U.S. Surface Coal Mines and Effective Land Reclamation, U.S. Bureau of Mines, Open File Report 74-75, Washington, D.C. (1975).

U.S. DOE Energy Information Administration, Bituminous Coal and Lignite Production and Mine Operations, DOE/EIA-0118.

Waage, Frenck, "The Arctangent Production Function and Its Dual, the Log-Tangent Cost Function," Rutgers University mimeograph (1977).

White, Halbert, "Nonlinear Regression on Cross-Section Data," Econometrica 48 (3) (1980), 721-746.

Zellner, Arnold, Jan Kmenta, and Jacques Drèze, "Specification and Estimation of Cobb-Douglas Production Function Models," Econometrica 34 (4) (1966), 784-795.

Zimmerman, Martin B., The U.S. Coal Industry: The Economics of Policy Choice (Cambridge, MA: MIT Press, 1981). 